\title{
Global Existence and Large Time Asymptotic Behavior of Strong Solution to the Cauchy Problem of 2D Density-Dependent Boussinesq Equations with Vacuum
}

\author{
Min Liu \\ College of Science, University of Shanghai for Science and Technology, Shanghai, China \\ Email: liumin4521@163.com
}

How to cite this paper: Liu, M. (2019) Global Existence and Large Time Asymptotic Behavior of Strong Solution to the Cauchy Problem of 2D Density-Dependent Boussinesq Equations with Vacuum. Journal of Applied Mathematics and Physics, 7, 2333-2351.

https://doi.org/10.4236/jamp.2019.710159

Received: September 12, 2019

Accepted: October 12, 2019

Published: October 15, 2019

Copyright $\odot 2019$ by author(s) and Scientific Research Publishing Inc. This work is licensed under the Creative Commons Attribution International License (CC BY 4.0).

http://creativecommons.org/licenses/by/4.0/

\begin{abstract}
We dedicate to the $2 \mathrm{D}$ density-dependent nonhomogeneous incompressible Boussinesq equations with vacuum on $\Omega \subset \mathbb{R}^{2}$. At infinity, if the attenuation of initial density and temperature is not very slow. And it is gained that there is a global strong solution and is unique for the 2D Cauchy problem with the initial density which can allow vacuum conditions and even have compact support. Besides, the large time decay rates of the gradients of velocity, temperature and pressure can also be obtained which are also the same as those of the homogeneous case.
\end{abstract}

\section{Keywords}

Non-Homogeneous Incompressible Boussinesq Equation, Strong Solution, Large Time Behavior, Existence and Uniqueness

\section{Introduction}

The Boussinessq equation is a coupling of the fluid temperature and velocity field. For this paper, we consider the Cauchy problem of 2D nonhomogeneous incompressible Boussinessq equations which read as follows:

$$
\left\{\begin{array}{l}
\rho_{t}+\operatorname{div}(\rho u)=0, \\
(\rho u)_{t}+\operatorname{div}(\rho u \otimes u)+\nabla p=\mu \Delta u, \quad x=\left(x_{1}, x_{2}\right) \in \Omega, t \geq 0, \\
\theta_{t}+u \cdot \nabla \theta-\kappa \Delta \theta=0, \\
\operatorname{div} u=0,
\end{array}\right.
$$

where $\rho=\rho(x, t)$ is the density, $u=\left(u^{1}, u^{2}\right)(x, t)$ represents the velocity, 
$p=p(x, t)$ stands for the pressure and $\theta=\theta(x, t)$ denotes the temperature of the fluid; $\mu>0$ is the viscosity coefficient; $\kappa>0$ is the thermal diffusivity.

The initial data is given by

$$
\rho(x, 0)=\rho_{0}(x), \theta(x, 0)=\theta_{0}(x), \rho u(x, 0)=\rho_{0} u_{0}(x), \quad x \in \mathbb{R}^{2} .
$$

The system (1.1) is a simple model widely used in the modeling of atmospheric motions and oceanic, and it plays an important role in the atmospheric sciences (see [1]). The Boussinessq equation is a coupling of the fluid velocity field and the temperature field. In particular, the $2 \mathrm{D}$ Boussinesq equations act as a lower-dimensional model of the $3 \mathrm{D}$ hydrodynamics equations and can be originated from the conservation laws of mass, energy and momentum (see [2]). The $2 \mathrm{D}$ Boussinesq equations can be seen as a special case of the $3 \mathrm{D}$ incompressible Navier-Stokes and Euler equations, with similar vortex stretching mechanism to 3D incompressible fluids. However, compared with the Navier-Stokes equation and the Euler equation, it has an unknown temperature function and there is a complex nonlinear relationship between the temperature function and the velocity and pressure (see [3] [4] [5] [6]). Moreover, the Boussinesq system (1.1) with $\theta=0$, there have been a lot of results. When $\rho_{0}>0$, many scholars have done a lot of research in recent years (Reference [4] [5] [7]).

In recent years, the Boussinesq system with $\rho>0$ has attracted the attention of many mathematicians, and many related research results have emerged. The study of viscous thermal diffusion Boussinesq equations, that is the system (1.1) with $\mu>0$ and $\kappa>0$, is popular. Lorca [8] and Boldrini [9] gained the existence of global weak solutions for Boussinesq equations with small initial values. And they also studied the existence of local strong solutions under general initial conditions. Recently, much attention has attracted by the density-dependent viscous Boussinesq equations. But, the regularity questions of the case of (1.1) with the initial data can be arbitrarily large, whic is an open problem. Qiu and Yao [10] showed the local existence and uniqueness of strong solutions of multi-dimensional incompressible density-dependent Boussinesq equations in Besov spaces. The paper [11] studied regularity criteria for three-dimensional incompressible density-dependent Boussinesq equations. However, there is little research on Boussinesq system when the initial density may include vacuum state or compact support. Because the initial density including the vacuum state will affect the temperature and pressure, the interaction among density, temperature and pressure will increase the non-linear coupling of the system (1.1), thus it may make the problem more complex. Recently, the global existence of strong solutions to the 2D Cauchy problem is given by Lü-Xu-Zhong [14], the related research refers to [12]-[17] [18]. Particularly, the initial density will include vacuum condition and one has compact support and the initial data could be arbitrarily large. Therefore, motivated by [14], we studied the Boussinesq system with initial density including vacuum and general big data initial. However, the divergence of temperature in the system (1.1) cannot be zero. This enhances the coupling of $u \cdot \nabla \theta$, and we need to solve some new difficulties. 
Now, we make some comments on the analysis of the key ingredients of this paper. If the local solution is extended to the global solution, we need to get global a priori estimates on strong solution to (1.1)-(1.2) in proper higher norms. Because of the strong coupling between temperature and velocity field, the $u \cdot \nabla \theta$ will give rise to some new difficulties. It seems difficult to bound the $L^{p}\left(\mathbb{R}^{2}\right)$-norm of $u$ in terms of $\left\|\rho^{1 / 2} u\right\|_{L^{2}\left(\mathbb{R}^{2}\right)}$ and $\|\nabla u\|_{L^{2}\left(\mathbb{R}^{2}\right)}$. In light of [15] [16] [19] [20], we try to estimate on the $L^{\infty}\left(0, T ; L^{2}\left(\mathbb{R}^{2}\right)\right)$-norm of $\nabla u$ and $\nabla \theta$, it can replace the usual $u_{t}$ with the $\dot{u} \triangleq u_{t}+u \cdot \nabla u$ to multiply by $(1.1)_{2}$ (see [21]). The most important thing is to control the term

$$
H_{2} \triangleq \int p \partial_{i} u^{j} \partial_{j} u^{i} \mathrm{~d} x
$$

According to [9] [12], since $\partial_{i} u^{j} \partial_{j} u^{i} \in \mathcal{H}^{1}$ and $p \in B M O$, we have the term $\|p\|_{\text {Вмо }}\left\|\partial_{j} u^{i} \partial_{i} u^{j}\right\|_{\mathcal{H}^{1}}$. And because $\operatorname{div}(\nabla u)=\nabla(\operatorname{div} u)=0$ and $\nabla^{\perp} \cdot\left(\nabla u^{j}\right)=0$ combined (2.6) and (2.8), the term $H_{2}$ in practice can be bounded by $\|\nabla p\|_{L^{2}}\|\nabla u\|_{L^{2}}^{2}$ (see (3.11)). Next, due to the strong coupled term $u \cdot \nabla \theta$, we cannot estimate directly the $L^{2}\left(\mathbb{R}^{2} \times(0, T)\right)$-norm of $\theta_{t}$. Because of multiplying $(1.1)_{3}$ by $\theta_{t}$, we can't get $L^{2}\left(\mathbb{R}^{2} \times(0, T)\right)$-norm of $\Delta \theta$ and there will be items related to time $t$. Thus, we can use the $\Delta \theta$ instead of the usual $\theta_{t}$ to multiply by $(1.1)_{3}$, and integration by parts that the coupled term $u \cdot \nabla \theta$ can be controlled (see (3.13)). Then, we apply the Stokes system to obtain the $L^{r}\left(\mathbb{R}^{2}\right)$ -norm of $\nabla^{2} u$ and the $L^{r}\left(\mathbb{R}^{2}\right)$-norm of $\nabla p$ (see (3.16)), and combined with the $\partial_{t}+u \cdot \nabla$ acts on $(1.1)_{2}^{j}$ and multiplied by $\dot{u}^{j}$ to get the (3.20) and make further efforts to give the (3.37) (see Lemma 3.3 and 3.5). In addition, it is sufficient to bound the $L^{p}\left(\mathbb{R}^{2}\right)$-norm of $\rho u$ instead of $u$. More precisely, using Lemma 2.4 (see (3.35)), reference [4], and along with the estimate of $\rho$ (see (3.36)), we can find the desired estimates on the $L^{p}\left(\mathbb{R}^{2}\right)$-norm of $\rho u$ (see (3.40)). Finally, we gain the $L^{2}$-norm of $\bar{x}^{1 / 2} \theta$ and $\bar{x}^{1 / 2} \nabla \theta$ (see (3.56)) and (see (3.57)), which are important to bound the $L^{2}\left(\mathbb{R}^{2} \times(0, T)\right)$-norm of both $t^{1 / 2} \nabla u_{t}$ and $t^{1 / 2} \nabla \theta_{t}$ and the $L^{\infty}\left(0, T ; L^{2}\left(\mathbb{R}^{2}\right)\right)$-norm of $t^{1 / 2} \nabla^{2} \theta$, see Lemma 3.8 .

Now, we go back to (1.1). it should be noted here that the notations and conventions employed throughout the paper. For $R>0$, set

$$
B_{R} \triangleq\left\{x \in \mathbb{R}^{2}|| x \mid<R\right\}, \quad \int f \mathrm{~d} x \triangleq \int_{\mathbb{R}^{2}} f \mathrm{~d} x .
$$

Furthermore, for $1 \leq r \leq \infty, k \geq 1$, we denote the standard Lebesgue and Sobolve spaces as follows:

$$
L^{r}=L^{r}\left(\mathbb{R}^{2}\right), \quad W^{k, r}=W^{k, r}\left(\mathbb{R}^{2}\right), \quad H^{r}=W^{k, 2} .
$$

Then, we will define precisely what mean by strong solution to (1.1) as follows:

Definition 1.1. (see [13]) If all derivatives related to (1.1) for $(\rho, u, p, \theta)$ are regular distributions, and system (1.1) satisfy almost everywhere in $\mathbb{R}^{2} \times(0, T)$, then $(\rho, u, p, \theta)$ is named a strong solution to (1.1).

In a general way, it can assume that $\rho_{0}$ holds 


$$
\int_{\mathbb{R}^{2}} \rho_{0} \mathrm{~d} x=1 .
$$

The (1.4) signifies that there is a positive constant $N_{0}$ such that

$$
\int_{B_{N_{0}}} \rho_{0} \mathrm{~d} x \geq \frac{1}{2} \int \rho_{0} \mathrm{~d} x=\frac{1}{2} .
$$

Theorem 1.1 In view of (1.4) and (1.5), it assumes that the initial data $\left(\rho_{0}, u_{0}, \theta_{0}\right)$ hold that for any given numbers $a>1$ and $q>2$,

$$
\left\{\begin{array}{l}
\rho_{0} \geq 0, \rho_{0} \bar{x}^{a} \in L^{1} \cap H^{1} \cap W^{1, q}, \nabla u_{0} \in L^{2}, \sqrt{\rho_{0}} u_{0} \in L^{2}, \\
\theta_{0} \geq 0, \theta_{0} \bar{x}^{\frac{a}{2}} \in L^{2}, \nabla \theta_{0} \in L^{2}, \operatorname{div} u_{0}=0,
\end{array}\right.
$$

where

$$
\bar{x} \triangleq\left(e+|x|^{2}\right)^{\frac{1}{2}} \log ^{2}\left(e+|x|^{2}\right) .
$$

In that way, it has a unique global strong solution $(\rho, u, p, \theta)$ for the problem (1.1)-(1.2) satisfying that for any $0<T<\infty$,

$$
\left\{\begin{array}{l}
0 \leq \rho \in C\left([0, T] ; L^{1} \cap H^{1} \cap W^{1, q}\right), \\
\rho \bar{x}^{a} \in L^{\infty}\left(0, T ; L^{1} \cap H^{1} \cap W^{1, q}\right), \\
\sqrt{\rho} u, \nabla u, \bar{x}^{-1} u, \sqrt{t} \sqrt{\rho} u_{t}, \sqrt{t} \nabla p, \sqrt{t} \nabla^{2} u \in L^{\infty}\left(0, T ; L^{2}\right), \\
\nabla \theta \in L^{2}\left(0, T_{1} ; H^{1}\right), \sqrt{t} \nabla u \in L^{2}\left(0, T ; W^{1, q}\right), \\
\theta, \theta \bar{x}^{a / 2}, \nabla \theta, \sqrt{t} \theta_{t}, \sqrt{t} \nabla^{2} \theta, \sqrt{t} \nabla \theta \bar{x}^{a / 2} \in L^{\infty}\left(0, T ; L^{2}\right), \\
\nabla u \in L^{2}\left(0, T ; H^{1}\right) \cap L^{(q+1) / q}\left(0, T ; W^{1, q}\right), \\
\nabla p \in L^{2}\left(0, T ; L^{2}\right) \cap L^{(q+1) / q}\left(0, T ; L^{q}\right), \\
\nabla \theta \in L^{2}\left(0, T ; H^{1}\right), \theta_{t}, \nabla \theta \bar{x}^{a / 2} \in L^{2}\left(0, T ; L^{2}\right), \\
\sqrt{t} \nabla u \in L^{2}\left(0, T ; W^{1, q}\right), \\
\sqrt{\rho} u_{t}, \sqrt{t} \nabla u_{t}, \sqrt{t} \nabla \theta_{t}, \sqrt{t} \bar{x}^{-1} u_{t} \in L^{2}\left(R^{2} \times(0, T)\right),
\end{array}\right.
$$

and

$$
\inf _{0 \leq t \leq T} \int_{B_{N_{1}}} \rho(x, t) \mathrm{d} x \geq \frac{1}{4}
$$

It's about positive constant $N_{1}$ depending only $\left\|\rho_{0}\right\|_{L^{2}},\left\|\rho_{0}^{\frac{1}{2}} u_{0}\right\|_{L^{2}}, \quad N_{0}$ and $T$. The $(\rho, u, p, \theta)$ has the following decay rates, that is for $t \geq 1$,

$$
\left\{\begin{array}{l}
\|\nabla u(\cdot, t)\|_{L^{2}}+\|\nabla \theta(\cdot, t)\|_{L^{2}} \leq C t^{-1 / 2}, \\
\left\|\nabla^{2} u(\cdot, t)\right\|_{L^{2}}+\|\nabla p(\cdot, t)\|_{L^{2}} \leq C t^{-1},
\end{array}\right.
$$

where $\mathrm{C}$ depends only on $\mu, \kappa,\left\|\rho_{0}\right\|_{L^{1} \cap L^{\infty}},\left\|\rho_{0}^{1 / 2} u_{0}\right\|_{L^{2}},\left\|\nabla u_{0}\right\|_{L^{2}}$, and $\left\|\theta_{0}\right\|_{H^{1}}$.

Remark 1.1 If the temperature function is zero, i.e., $\theta=0$, then (1.1) is the 
well-known Navier-Stokes equations, and Theorem 1.1 is the same as those results of [12].

Remark 1.2 Theorem 1.1 goes for arbitrarily large initial data, it can also find the global strong solutions to the 2D incompressible Boussinesq equations with the smallness condition on the initial energy see [8] [9].

In next section, we shall first state some basic truths and inequalities. Those things will be employed later in this paper. In the last section is committed to some priori estimates and prove the theorem 1.1.

\section{Preliminaries}

For the section, we will recall some known truths and elementary inequalities, which will be used frequently later. Then for initial data, it assumes that there is a unique local strong solution. As follows:

Lemma 2.1 see [21] Assume that $\left(\rho_{0}, u_{0}, \theta_{0}\right)$ satisfies (1.6). Then there exists a small time $T_{0}>0$ and a unique strong solution $(\rho, u, p, \theta)$ to the problem (1.1)-(1.2) in $\mathbb{R}^{2} \times\left(0, T_{0}\right)$ satisfying (1.8) and (1.9).

Lemma 2.2 (see ([22] Theorem 1.1)) (Galiardo-Nirenberg). For $m \in[2, \infty)$, $q \in(1, \infty)$, and $r \in(2, \infty)$, there exists some generic constant $C>0$ which may relay on $m, q$, and $r$ such that for $f \in H^{1}\left(\mathbb{R}^{2}\right)$ and $g \in L^{q}\left(\mathbb{R}^{2}\right) \cap D^{1, r}\left(\mathbb{R}^{2}\right)$, we have

$$
\begin{gathered}
\|f\|_{L^{m}\left(\mathbb{R}^{2}\right)}^{m} \leq C\|f\|_{L^{2}\left(\mathbb{R}^{2}\right)}^{2}\|\nabla f\|_{L^{2}\left(\mathbb{R}^{2}\right)}^{m-2}, \\
\|g\|_{C\left(\overline{\mathbb{R}^{2}}\right)} \leq C\|g\|_{L^{q}\left(\mathbb{R}^{2}\right)}^{q(r-2) /(2 r+q(r-2))}\|\nabla g\|_{L^{\prime}\left(\mathbb{R}^{2}\right)}^{2 r /(2 r+q(r-2))} .
\end{gathered}
$$

The following weighted $L^{n}$ bounds for elements in $\tilde{D}^{1,2}\left(\mathbb{R}^{2}\right) \triangleq\left\{v \in H_{\text {loc }}^{1}\left(\mathbb{R}^{2}\right) \mid \nabla v \in L^{2}\left(\mathbb{R}^{2}\right)\right\}$ can be found in ([22], Theorem 1.1).

Lemma 2.3 (see ([4], TheoremB.1)) For $h \in[2, \infty)$ and $\lambda \in(1+h / 2, \infty)$, there exists a positive constant $C$ such that for all $v \in \tilde{D}^{1,2}\left(\mathbb{R}^{2}\right)$,

$$
\left(\int_{\mathbb{R}^{2}} \frac{|v|^{h}}{e+|x|^{2}}\left(\log \left(e+|x|^{2}\right)\right)^{-\lambda} \mathrm{d} x\right)^{1 / h} \leq C\|v\|_{L^{2}\left(B_{1}\right)}+C\|\nabla v\|_{L^{2}\left(\mathbb{R}^{2}\right)} .
$$

The Lemma 2.3 combined with the Poincaré inequality gets the following useful results on weighted bounds, we can also refer to ([21] Lemma 2.4).

Lemma 2.4 (see ([23] Lemma 2.3)) We can refer to $\bar{x}$ in (1.6), and assume that $\rho \in L^{1}\left(\mathbb{R}^{2}\right) \cap L^{\infty}\left(\mathbb{R}^{2}\right)$ is a non-negative function such that

$$
\|\rho\|_{L^{1}\left(B_{N_{0}}\right)} \geq M_{0}, \quad\|\rho\|_{L^{1}\left(\mathbb{R}^{2}\right) \cap L^{\infty}\left(\mathbb{R}^{2}\right)} \leq M_{1},
$$

for positive constants $M_{0}, M_{1}$, and $N_{0} \geq 1$ with $B_{N_{0}} \subset \mathbb{R}^{2}$. Then for $\alpha>0$, $\beta>0$, there is a positive constant $C$ depending only on $\alpha, \beta, M_{0}, M_{1}$, and $N_{0}$ such that every $v \in \tilde{D}^{1,2}\left(\mathbb{R}^{2}\right)$ satisfies

$$
\left\|v \bar{X}^{-\beta}\right\|_{L(2+\alpha) / \tilde{\beta}\left(\mathbb{R}^{2}\right)} \leq C\left\|\rho^{1 / 2} v\right\|_{L^{2}\left(\mathbb{R}^{2}\right)}+C\|\nabla v\|_{L^{2}\left(\mathbb{R}^{2}\right)},
$$

with $\tilde{\beta}=\min \{1, \beta\}$. 
Finally, let $\mathrm{BMO}\left(\mathbb{R}^{2}\right)$ and $\mathcal{H}^{1}\left(\mathbb{R}^{2}\right)$ represent BMO and Hardy spaces (see [24], chapter 4). In the next section, some facts are more important to prove the lemma 3.2.

Lemma 2.5 (see ([25] TheoremII.1)) (i) There is a positive constant $C$ such that

$$
\|G \cdot M\|_{\mathcal{H}^{1}\left(\mathbb{R}^{2}\right)} \leq C\|G\|_{L^{2}\left(\mathbb{R}^{2}\right)}\|M\|_{L^{2}\left(\mathbb{R}^{2}\right)},
$$

for all $G \in L^{2}\left(\mathbb{R}^{2}\right)$ and $M \in L^{2}\left(\mathbb{R}^{2}\right)$ satisfying

$$
\operatorname{div} G=0, \quad \nabla^{\perp} \cdot M=0 \quad \text { in } D^{\prime}\left(\mathbb{R}^{2}\right) .
$$

(ii) There is a positive constant $C$ such that

$$
\|f\|_{\mathrm{BMO}\left(\mathbb{R}^{2}\right)} \leq C\|\nabla f\|_{L^{2}\left(\mathbb{R}^{2}\right)},
$$

for all $f \in \tilde{D}^{1,2}\left(\mathbb{R}^{2}\right)$.

Proof. (i) Please refer to ([25] Theorem II.1) for detailed proof.

(ii) The follows together with the Poincare inequality that for any ball $B \subset\left(\mathbb{R}^{2}\right)$

$$
\frac{1}{|B|} \int_{B}\left|v(x)-\frac{1}{|B|} \int_{B} v(y) \mathrm{d} y\right| \mathrm{d} x \leq C\left(\int_{B}|\nabla v|^{2} \mathrm{~d} x\right)^{1 / 2},
$$

which directly gives (2.8).

\section{Convergence Rate of the Solution}

\subsection{Lower Order Estimates}

Due to $\operatorname{divu}=0$, it will estimate the $L^{\infty}\left(0, T ; L^{r}\right)$-norm of $\rho$, as follows:

Lemma 3.1 (see [4]) There exists a positive constant $C$ depending only on $\left\|\rho_{0}\right\|_{L^{1} \cap L^{\infty}}$ such that

$$
\sup _{t \in[0, T]}\|\rho\|_{L^{1} \cap L^{\infty}} \leq C .
$$

Then, we will estimate the $L^{\infty}\left(0, T ; L^{2}\right)$-norm of $\nabla \theta$ and $\nabla u$.

Lemma 3.2. There is a positive constant $C$ depending only on $\mu, \kappa$, $\left\|\rho_{0}\right\|_{L^{\infty}},\left\|\nabla u_{0}\right\|_{L^{2}},\left\|\sqrt{\rho_{0}} u_{0}\right\|_{L^{2}}$, and $\left\|\theta_{0}\right\|_{H^{1}}$ such that

$$
\sup _{t \in[0, T]}\left(\|\nabla u\|_{L^{2}}^{2}+\|\nabla \theta\|_{L^{2}}^{2}+\|\theta\|_{L^{2}}^{2}\right)+\int_{0}^{T}\left(\|\sqrt{\rho} \dot{u}\|_{L^{2}}^{2}+\|\Delta \theta\|_{L^{2}}^{2}\right) \mathrm{d} t \leq C,
$$

Here $\dot{u} \triangleq \partial_{t} u+u \cdot \nabla u$, and have

$$
\sup _{t \in[0, T]} t\left(\|\nabla u\|_{L^{2}}^{2}+\|\nabla \theta\|_{L^{2}}^{2}\right)+\int_{0}^{T} t\left(\|\sqrt{\rho} \dot{u}\|_{L^{2}}^{2}+\|\Delta \theta\|_{L^{2}}^{2}\right) \mathrm{d} t \leq C .
$$

Proof. Invoking standard energy estimate, multiplying $(1.1)_{2}$ by $u$ and integrating the resulting equality over $\mathbb{R}^{2}$, we get

$$
\sup _{t \in[0, T]}\|\sqrt{\rho} u\|_{L^{2}}^{2}+\int_{0}^{T}\|\nabla u\|_{L^{2}}^{2} \mathrm{~d} t \leq C .
$$

Multiplying $(1.1)_{3}$ by $\theta$ and integrating the resulting equality over $\mathbb{R}^{2}$, we have

$$
\sup _{t \in[0, T]}\|\theta\|_{L^{2}}^{2}+\int_{0}^{T}\|\nabla \theta\|_{L^{2}}^{2} \mathrm{~d} t \leq C
$$


The (3.4) combined with (3.5) that gives

$$
\sup _{t \in[0, T]}\left(\|\sqrt{\rho} u\|_{L^{2}}^{2}+\|\theta\|_{L^{2}}^{2}\right)+\int_{0}^{T}\left(\|\nabla u\|_{L^{2}}^{2}+\|\nabla \theta\|_{L^{2}}^{2}\right) \mathrm{d} t \leq C .
$$

Next, multiplying $(1.1)_{2}$ by $\dot{u}$ and integrating the resulting equality over $\mathbb{R}^{2}$, we have

$$
\int \rho|\dot{u}|^{2} \mathrm{~d} x=\int \mu \Delta u \cdot \dot{u} \mathrm{~d} x-\int \nabla p \cdot \dot{u} \mathrm{~d} x \triangleq H_{1}+H_{2} .
$$

Then we can follow form integrating $H_{i}(i=1,2)$ by parts and $(2.1)$ that

$$
\begin{aligned}
H_{1} & =\mu \int \Delta u \cdot\left(u_{t}+u \cdot \nabla u\right) \mathrm{d} x \\
& =-\frac{\mu}{2} \frac{\mathrm{d}}{\mathrm{d} t}\|\nabla u\|_{L^{2}}^{2}-\mu \int \partial_{i} u^{j} \partial_{i}\left(u^{k} \partial_{k} u^{j}\right) \mathrm{d} x \\
& \leq-\frac{\mu}{2} \frac{\mathrm{d}}{\mathrm{d} t}\|\nabla u\|_{L^{2}}^{2}+C\|\nabla u\|_{L^{3}}^{3} \\
& \leq-\frac{\mu}{2} \frac{\mathrm{d}}{\mathrm{d} t}\|\nabla u\|_{L^{2}}^{2}+C\|\nabla u\|_{L^{2}}^{2}\left\|\nabla^{2} u\right\|_{L^{2}} .
\end{aligned}
$$

Integration by parts together with $(1.1)_{4}$ gives

$$
H_{2}=-\int \nabla p\left(u_{t}+u \cdot \nabla u\right) \mathrm{d} x=\int p \partial_{j} u^{i} \partial_{i} u^{j} \mathrm{~d} x \leq C\|p\|_{\text {BMO }}\left\|\partial_{j} u^{i} \partial_{i} u^{j}\right\|_{\mathcal{H}^{1}},
$$

For the last inequality, because of the duality of $\mathcal{H}^{1}$ space and BMO (see ([27] Chapter IV)). And $\operatorname{div}\left(\partial_{j} u\right)=\partial_{j} \operatorname{divu}=0, \nabla^{\perp} \cdot\left(\nabla u^{j}\right)=0$, and (2.6) yields

$$
\left\|\partial_{j} u^{i} \partial_{i} u^{j}\right\|_{\mathcal{H}^{1}} \leq C\|\nabla u\|_{L^{2}}\|\nabla u\|_{L^{2}} \text {. }
$$

The (3.9) combined with (3.10) and (2.8) gives

$$
\left|H_{2}\right|=\left|\int p \partial_{j} u^{i} \partial_{i} u^{j} \mathrm{~d} x\right| \leq C\|p\|_{B M O}\|\nabla u\|_{L^{2}}^{2} \leq C\|\nabla p\|_{L^{2}}\|\nabla u\|_{L^{2}}^{2} .
$$

Next, substituting (3.8) and (3.11) into (3.7) gives

$$
\frac{\mu}{2} \frac{\mathrm{d}}{\mathrm{d} t}\|\nabla u\|_{L^{2}}^{2}+\|\sqrt{\rho} \dot{u}\|_{L^{2}}^{2} \leq C\left(\left\|\nabla^{2} u\right\|_{L^{2}}+\|\nabla p\|_{L^{2}}\right)\|\nabla u\|_{L^{2}}^{2} .
$$

Then, the $(1.1)_{3}$ multiplied by $\Delta \theta$ and integrating the resulting equality by parts over $\mathbb{R}^{2}$, and together with Hölder's and (11) that

$$
\begin{aligned}
& \frac{\mathrm{d}}{\mathrm{d} t} \int|\nabla \theta|^{2} \mathrm{~d} x+2 \kappa \int|\Delta \theta|^{2} \mathrm{~d} x \\
& \leq C \int|\nabla u \| \nabla \theta|^{2} \mathrm{~d} x \\
& \leq C\|\nabla u\|_{L^{3}}\|\nabla \theta\|_{L^{2}}^{\frac{4}{3}}\|\nabla \theta\|_{L^{2}}^{\frac{2}{3}} \\
& \leq C\|\nabla u\|_{L^{2}}^{2}\left\|\nabla^{2} u\right\|_{L^{2}}+C\|\nabla \theta\|_{L^{2}}^{4}+C\|\Delta \theta\|_{L^{2}}^{2},
\end{aligned}
$$

which combined with (3.12) and (3.6) gives

$$
\begin{aligned}
& \frac{\mathrm{d}}{\mathrm{d} t}\left(\frac{\mu}{2}\|\nabla u\|_{L^{2}}^{2}+\|\nabla \theta\|_{L^{2}}^{2}\right)+\|\sqrt{\rho} \dot{u}\|_{L^{2}}^{2}+2 \kappa\|\Delta \theta\|_{L^{2}}^{2} \\
& \leq C\|\nabla \theta\|_{L^{2}}^{4}+C\left(\left\|\nabla^{2} u\right\|_{L^{2}}+\|\nabla p\|_{L^{2}}\right)\|\nabla u\|_{L^{2}}^{2} .
\end{aligned}
$$

Due to $(\rho, u, p, \theta)$ solves the following Stokes system see [26] 


$$
\begin{cases}-\mu \Delta u+\nabla p=-\rho \dot{u}, & x \in \mathbb{R}^{2}, \\ \operatorname{div} u=0, & x \in \mathbb{R}^{2}, \\ u(x) \rightarrow 0, & |x| \rightarrow \infty,\end{cases}
$$

Using the standard $L^{r}$-estimate to (3.15) holds that for any $r>1$,

$$
\left\|\nabla^{2} u\right\|_{L^{r}}+\|\nabla p\|_{L^{r}} \leq C\|\rho \dot{u}\|_{L^{r}} \leq C\|\sqrt{\rho} \dot{u}\|_{L^{r}} .
$$

(3.14) combined with and (3.16) gives

$$
\begin{aligned}
& \frac{\mathrm{d}}{\mathrm{d} t}\left(\frac{\mu}{2}\|\nabla u\|_{L^{2}}^{2}+\|\nabla \theta\|_{L^{2}}^{2}\right)+\|\sqrt{\rho} \dot{u}\|_{L^{2}}^{2}+2 \kappa\|\Delta \theta\|_{L^{2}}^{2} \\
& \leq C\|\nabla \theta\|_{L^{2}}^{4}+C\|\nabla u\|_{L^{2}}^{4}+\varepsilon\|\sqrt{\rho} \dot{u}\|_{L^{2}}^{2},
\end{aligned}
$$

where $\varepsilon$ is to be determined. Choosing $\varepsilon=\frac{1}{2}$, it follows from (3.6) and (3.17) that

$$
\begin{aligned}
& \frac{\mathrm{d}}{\mathrm{d} t}\left(\frac{\mu}{2}\|\nabla u\|_{L^{2}}^{2}+\|\nabla \theta\|_{L^{2}}^{2}\right)+\frac{1}{2}\|\sqrt{\rho} \dot{u}\|_{L^{2}}^{2}+2 \kappa\|\Delta \theta\|_{L^{2}}^{2} \\
& \leq C\left(\|\nabla u\|_{L^{2}}^{2}+\|\nabla \theta\|_{L^{2}}^{2}\right)\left(\|\nabla \theta\|_{L^{2}}^{2}+\|\nabla u\|_{L^{2}}^{2}+C\right) .
\end{aligned}
$$

the (3.18) together with (3.6), (3.17) and (2.1) gives (3.2). Then, (3.17) multiplied by $\mathrm{t}$, we have

$$
\begin{aligned}
& \frac{\mathrm{d}}{\mathrm{d} t} t\left(\frac{\mu}{2}\|\nabla u\|_{L^{2}}^{2}+\|\nabla \theta\|_{L^{2}}^{2}\right)-\frac{\mu}{2}\|\nabla u\|_{L^{2}}^{2}-\|\nabla \theta\|_{L^{2}}^{2}+\frac{1}{2} t\|\sqrt{\rho} \dot{u}\|_{L^{2}}^{2}+2 \kappa t\|\Delta \theta\|_{L^{2}}^{2} \\
& \leq t\left(\|\nabla u\|_{L^{2}}^{2}+\|\nabla \theta\|_{L^{2}}^{2}\right)\left(\|\nabla \theta\|_{L^{2}}^{2}+\|\nabla u\|_{L^{2}}^{2}+C\right),
\end{aligned}
$$

the (3.19) combined Gronwall's inequality with (3.6) gives (3.3). Finally, it finishes the proof of lemma 3.2.

Lemma 3.3 There is some positive constant $C$ depending only on $\mu, \kappa$, $\left\|\rho_{0}\right\|_{L^{1} \cap L^{\infty}},\left\|\nabla u_{0}\right\|_{L^{2}}$, and $\left\|\rho_{0}^{\frac{1}{2}} u_{0}\right\|_{L^{2}}$ such that for $i=1,2$,

$$
\sup _{t \in[0, T]} t^{i}\|\sqrt{\rho} \dot{u}\|_{L^{2}}^{2}+\int_{0}^{T} t^{i}\|\nabla \dot{u}\|_{L^{2}}^{2} \mathrm{~d} t \leq C,
$$

and

$$
\sup _{t \in[0, T]} t^{i}\left(\left\|\nabla^{2} u\right\|_{L^{2}}^{2}+\|\nabla p\|_{L^{2}}^{2}\right) \leq C .
$$

Proof: Using $\partial_{t}+u \cdot \nabla$ to $(1.1)_{2}^{j}$, it follows from a few simple calculations that

$$
\begin{aligned}
& \partial_{t}\left(\rho \dot{u}^{j}\right)+\operatorname{div}\left(\rho u \dot{u}^{j}\right)-\mu \Delta \dot{u}^{j} \\
& =-\mu \partial_{i}\left(\partial_{i} u \cdot \nabla u^{j}\right)-\mu \operatorname{div}\left(\partial_{i} u \partial_{i} u^{j}\right)-\partial_{j} \partial_{t} p-(u \cdot \nabla) \partial_{j} p .
\end{aligned}
$$

Next, (3.22) multiplied by $\dot{u}^{j}$, and integration by parts and (1.1) $)_{4}$, we get 


$$
\begin{aligned}
& \frac{1}{2} \frac{\mathrm{d}}{\mathrm{d} t}\|\sqrt{\rho} \dot{u}\|_{L^{2}}^{2}+\mu\|\nabla \dot{u}\|_{L^{2}}^{2} \\
& =-\int \mu \partial_{i}\left(\partial_{i} u \cdot \nabla u^{j}\right) \dot{u}^{j} \mathrm{~d} x-\int \mu \operatorname{div}\left(\partial_{i} u \partial_{i} \dot{u}^{j}\right) \dot{u}^{j} \mathrm{~d} x \\
& \quad-\int\left(\dot{u}^{j} \partial_{t} \partial_{j} p+\dot{u}^{j}(u \cdot \nabla) \partial_{j} p\right) \mathrm{d} x \\
& \triangleq \sum_{i=1}^{3} M_{i} .
\end{aligned}
$$

Following the same argument as ([12] Lemma 3.3) we have the estimates of $\left(M_{i}(i=1,2,3)\right)$ as

$$
\sum_{i=1}^{3} M_{i} \leq \frac{\mathrm{d}}{\mathrm{d} t} \int p \partial_{j} u^{i} \partial_{i} u^{j} \mathrm{~d} x+C\left(\|p\|_{L^{4}}^{4}+\|\nabla u\|_{L^{4}}^{4}\right)+\frac{\mu}{2}\|\nabla \dot{u}\|_{L^{2}}^{2} .
$$

Substituting (3.24) into (3.23) gives

$$
\begin{aligned}
& \frac{\mathrm{d}}{\mathrm{d} t}\left(\frac{1}{2}\|\sqrt{\rho} \dot{u}\|_{L^{2}}^{2}-\int p \partial_{j} u^{i} \partial_{i} u^{j} \mathrm{~d} x\right)+\frac{\mu}{2}\|\nabla \dot{u}\|_{L^{2}}^{2} \\
& \leq C\|p\|_{L^{4}}^{4}+C\|\nabla u\|_{L^{4}}^{4} .
\end{aligned}
$$

For the left of (3.25), we have

$$
\begin{aligned}
\left|\int p \partial_{j} u^{i} \partial_{i} u^{j} \mathrm{~d} x\right| & \leq C\|\nabla p\|_{L^{2}}\|\nabla u\|_{L^{2}}^{2} \\
& \leq C\left(\left\|\nabla^{2} u\right\|_{L^{2}}+\|\nabla p\|_{L^{2}}\right)\|\nabla u\|_{L^{2}}^{2} \\
& \leq C\left\|\rho^{\frac{1}{2}} \dot{u}\right\|_{L^{2}}^{2}+C\|\nabla u\|_{L^{2}}^{4} .
\end{aligned}
$$

For the right of (3.25), (3.11) together with (3.16), (3.1) and Sobolev's inequality that

$$
\begin{aligned}
\|p\|_{L^{4}}^{4}+\|\nabla u\|_{L^{4}}^{4} & \leq C\left(\|\nabla p\|_{L^{\frac{4}{3}}}^{4}+\left\|\nabla^{2} u\right\|_{L^{\frac{4}{3}}}^{4}\right) \leq C\|\rho \dot{u}\|_{L^{\frac{4}{3}}}^{4} \\
& \leq C\|\rho\|_{L^{2}}^{2}\|\sqrt{\rho} \dot{u}\|_{L^{2}}^{4} \leq C\|\sqrt{\rho} \dot{u}\|_{L^{2}}^{4} .
\end{aligned}
$$

Substituting (3.26) and (3.27) into (3.25)

$$
\frac{\mathrm{d}}{\mathrm{d} t}\|\sqrt{\rho} \dot{u}\|_{L^{2}}^{2}+\frac{\mu}{2}\|\nabla \dot{u}\|_{L^{2}}^{2} \leq C\|\sqrt{\rho} \dot{u}\|_{L^{2}}^{4} .
$$

Next, multiplying (3.28) by $t^{i}(i=1,2)$, it follows from (3.3) and (3.6) that

$$
\frac{\mathrm{d}}{\mathrm{d} t} t^{i}\|\sqrt{\rho} \dot{u}\|_{L^{2}}^{2}-i t^{i-1}\|\sqrt{\rho} \dot{u}\|_{L^{2}}^{2}+\frac{\mu}{2}\|\nabla \dot{u}\|_{L^{2}}^{2} \leq C t^{i}\|\sqrt{\rho} \dot{u}\|_{L^{2}}^{2}\left(\|\sqrt{\rho} \dot{u}\|_{L^{2}}^{2}+C\right) \text {. }
$$

Then, the (3.29) along with Gronwall's inequality gives

$$
\sup _{t \in[0, T]} t^{i}\|\sqrt{\rho} \dot{u}\|_{L^{2}}^{2}+\int_{0}^{T} t^{i}\|\nabla \dot{u}\|_{L^{2}}^{2} \mathrm{~d} t \leq \int_{0}^{T} i t^{i-1}\|\sqrt{\rho} \dot{u}\|_{L^{2}}^{2} \mathrm{~d} t .
$$

Finally, due to $i=1,2$, it deduces from (3.3) to lead to (3.20). The (3.21) is a direct consequence of (3.20) and (3.16). We will finish the proof of Lemma 3.3.

\subsection{Higher Order Estimates}

It concerns with the estimates on the higher-order derivatives of the strong solu- 
tion $(\rho, u, p, \theta)$ as follow:

Lemma 3.4 For a positive constant $C$ depending only on $\mu,\left\|\rho_{0}\right\|_{L^{1} \cap L^{\infty}}$, $\left\|\nabla u_{0}\right\|_{L^{2}},\left\|\rho_{0}^{\frac{1}{2}} u_{0}\right\|_{L^{2}},\left\|\rho_{0} \bar{x}^{a}\right\|_{L^{1}}, N_{0}$, and $T$, such that

$$
\sup _{t \in[0, T]}\left\|\rho \bar{X}^{a}\right\|_{L^{1}} \leq C(T) .
$$

Proof. For $M>1$, let $\varphi_{M} \in C_{0}^{\infty}\left(B_{M}\right)$ satisfy

$$
0 \leq \varphi_{M} \leq 1, \varphi_{M}(x)=\left\{\begin{array}{ll}
1, & |x| \leq M / 2, \\
0, & |x| \geq M,
\end{array} \quad\left|\nabla \varphi_{M}\right| \leq C M^{-1} .\right.
$$

It combines with (1.1) that

$$
\frac{\mathrm{d}}{\mathrm{d} t} \int \rho \varphi_{M} \mathrm{~d} x=\int \rho u \cdot \nabla \varphi_{M} \mathrm{~d} x \geq-C M^{-1}\left(\int \rho \mathrm{d} x\right)^{1 / 2}\left(\int \rho|u|^{2} \mathrm{~d} x\right)^{1 / 2} \geq-\tilde{C} M^{-1},
$$

in the last inequality of (3.33), it has applied (3.1) and (3.6). Integrating (3.33) and letting $M=N_{1} \triangleq 2 N_{0}+4 \tilde{C} T$, we obtain after using (1.5) that

$$
\begin{aligned}
\inf _{t \in[0, T]} \int_{B_{N_{1}}} \rho \mathrm{d} x & \geq \inf _{t \in[0, T]} \int \rho \varphi_{N_{1}} \mathrm{~d} x \geq \int \rho_{0} \varphi_{N_{1}} \mathrm{~d} x-\tilde{C} N_{1}^{-1} T \\
& \geq \int_{B_{N_{0}}} \rho_{0} \mathrm{~d} x-\frac{\tilde{C} T}{2 N_{0}+4 \tilde{C} T} \geq \frac{1}{4} .
\end{aligned}
$$

the (3.34) along with (3.1), (2.2), (3.6) and (3.2) that for any $\eta \in(0,1]$ and any $s>2$,

$$
\left\|u \bar{x}^{-\eta}\right\|_{L^{s / \eta}} \leq C\left(\left\|\rho^{1 / 2} u\right\|_{L^{2}}+\|\nabla u\|_{L^{2}}\right) \leq C .
$$

$(1.1)_{1}$ multiplied by $\bar{x}^{a}$ and integrating the resulting equality by parts over $\mathbb{R}^{2}$ find that

$$
\begin{aligned}
\frac{\mathrm{d}}{\mathrm{d} t} \int \rho \bar{x}^{a} \mathrm{~d} x & \leq C \int \rho|u| \bar{x}^{a-1} \log ^{2}\left(e+|x|^{2}\right) \mathrm{d} x \\
& \leq C\left\|\rho \bar{x}^{a-1+\frac{8}{8+a}}\right\|_{L_{L^{7+a}}}\left\|u \bar{x}^{-\frac{1}{8+a}}\right\|_{L^{8+a}} \\
& \leq C \int \rho \bar{x}^{a} \mathrm{~d} x+C .
\end{aligned}
$$

using the Gronwall's inequality to (3.36) gives (3.31) and it proves the lemma 3.4.

Lemma 3.5 There is a positive constant $C$ depending on $T$ such that

$$
\begin{aligned}
& \sup _{t \in[0, T]}\|\rho\|_{H^{1} \cap W^{1, q}}+\int_{0}^{T}\left(\left\|\nabla^{2} u\right\|_{L^{2}}^{2}+\left\|\nabla^{2} u\right\|_{L^{q}}^{\frac{q+1}{q}}+t\left\|\nabla^{2} u\right\|_{L^{2} \cap L^{q}}^{2}\right) \mathrm{d} t \\
& +\int_{0}^{T}\left(\|\nabla p\|_{L^{2}}^{2}+\|\nabla p\|_{L^{q}}^{\frac{q+1}{q}}+t\|\nabla p\|_{L^{2} \cap L^{q}}^{2}\right) \mathrm{d} t \leq C(T) .
\end{aligned}
$$

Proof. We can follow from the $(1.1)_{1}$ that $\nabla \rho$ holds for any $r \geq 2$,

$$
\frac{\mathrm{d}}{\mathrm{d} t}\|\nabla \rho\|_{L^{r}} \leq C(r)\|\nabla u\|_{L^{\infty}}\|\nabla \rho\|_{L^{r}} .
$$


Next, employing Lemma 2.2, (3.2) and (3.16), we have $q>2$,

$$
\|\nabla u\|_{L^{\infty}} \leq C\|\nabla u\|_{L^{2}}^{\frac{q-2}{2(q-1)}}\left\|\nabla^{2} u\right\|_{L^{q}}^{\frac{q}{2(q-1)}} \leq C\|\rho \dot{u}\|_{L^{q}}^{\frac{q}{2(q-1)}} .
$$

It follows from (3.34), (3.1), (2.2) and (3.31) that for any $s>2$,

$$
\begin{aligned}
\|\rho v\|_{L^{s}} & \leq C\left\|\rho \bar{x}^{\frac{3 a}{4 s}}\right\|_{L^{\frac{4 s}{3}}}\left\|v \bar{x}^{-\frac{3 a}{4 s}}\right\|_{L^{4 s}} \\
& \leq C\|\rho\|_{L^{\infty}}^{\frac{4 s-3}{4 s}}\left\|\rho \bar{x}^{a}\right\| \frac{3}{4 s}\left(\left\|\rho^{1 / 2} v\right\|_{L^{2}}+\|\nabla v\|_{L^{2}}\right) \\
& \leq C\left(\left\|\rho^{1 / 2} v\right\|_{L^{2}}+\|\nabla v\|_{L^{2}}\right),
\end{aligned}
$$

the (3.40) combine with the Gagliardo-Nirenberg inequality shows that

$$
\begin{aligned}
\|\rho \dot{u}\|_{L^{q}} & \leq C\|\rho \dot{u}\|_{L^{2}}^{\frac{2(q-1)}{q^{2}-2}}\|\rho \dot{u}\|_{L^{q^{2}}}^{\frac{q(q-2)}{q^{2}-2}} \\
& \leq C\|\rho \dot{u}\|_{L^{2}}^{\frac{2(q-1)}{q^{2}-2}}\left(\|\sqrt{\rho} \dot{u}\|_{L^{2}}+\|\nabla \dot{u}\|_{L^{2}}\right)^{\frac{q(q-2)}{q^{2}-2}} \\
& \leq C\left(\|\sqrt{\rho} \dot{u}\|_{L^{2}}+\|\sqrt{\rho} \dot{u}\|_{L^{2}}^{\frac{2(q-1)}{q^{2}-2}}\|\nabla \dot{u}\|_{L^{2}}^{\frac{q(q-2)}{q^{2}-2}}\right),
\end{aligned}
$$

which is deformed and calculated appropriately leads to

$$
\begin{aligned}
& \int_{0}^{T}\|\rho \dot{u}\|_{L^{q}}^{\frac{q+1}{q}} \mathrm{~d} t \\
& \leq C \int_{0}^{T}\|\sqrt{\rho} \dot{u}\| \|_{L^{2}}^{\frac{q+1}{q}} \mathrm{~d} t+\sup _{t \in[0, T]}\left(t\|\sqrt{\rho} \dot{u}\|_{L^{2}}^{2}\right)^{\frac{q^{2}-1}{2 q\left(q^{2}-2\right)}} \int_{0}^{T} t^{-\frac{q^{3}+q^{2}-2 q-2}{2 q\left(q^{2}-2\right)}}\left(t\|\nabla \dot{u}\|_{L^{2}}^{2}\right)^{\frac{q(q-2)(q+1)}{2 q\left(q^{2}-2\right)}} \mathrm{d} t \\
& \leq C \int_{0}^{T}\|\sqrt{\rho} \dot{u}\|_{L^{2}}^{2} \mathrm{~d} t+C \int_{0}^{T} t t^{-\frac{q^{3}+q^{2}-2 q-2}{q^{3}+q^{2}-2 q}} \mathrm{~d} t+C \int_{0}^{T} t\|\nabla \dot{u}\|_{L^{2}}^{2} \mathrm{~d} t \\
& \leq C \text {, } \\
& \int_{0}^{T} t\|\rho \dot{u}\|_{L^{q}}^{2} \mathrm{~d} t \leq C\left(\int_{0}^{T} t\|\nabla \dot{u}\|_{L^{2}}^{2} \mathrm{~d} t+1\right) \leq C .
\end{aligned}
$$

Then, the (3.42) and (3.39) implies

$$
\int_{0}^{T}\|\nabla u\|_{L^{\infty}} \mathrm{d} t \leq C
$$

Next, using Gronwall's inequality to (3.38) shows

$$
\sup _{t \in[0, T]}\|\nabla \rho\|_{L^{2} \cap L^{q}} \leq C .
$$

Then, letting $r=2$ in (3.16) and integrating the resulting equality over $[0, T]$, we obtain after using $(3.1),(3.2)$ and (3.3) that

$$
\int_{0}^{T}\left\|\nabla^{2} u\right\|_{L^{2}}^{2} \mathrm{~d} t+\int_{0}^{T}\|\nabla p\|_{L^{2}}^{2} \mathrm{~d} t \leq C
$$

Similarly, setting $r=q$ in (3.16) and integrating the resulting equality over $[0, T]$, we deduce from using (3.42), (3.1), (3.2) and (3.3) that 


$$
\int_{0}^{T}\left\|\nabla^{2} u\right\|_{L^{q}}^{\frac{q+1}{q}} \mathrm{~d} t+\int_{0}^{T}\|\nabla p\|_{L^{q}}^{\frac{q+1}{q}} \mathrm{~d} t \leq C .
$$

Multiplying (3.16) by $t$ and integrating the resulting equality over $[0, T]$, it can obtain after using (3.43), (3.1), (3.2) and (3.3) that

$$
\int_{0}^{T} t\left\|\nabla^{2} u\right\|_{L^{2} \cap L^{q}}^{2} \mathrm{~d} t+\int_{0}^{T} t\|\nabla p\|_{L^{2} \cap L^{q}}^{2} \mathrm{~d} t \leq C .
$$

Moreover, it can get from (3.46), (3.47) and (3.48) that

$$
\begin{aligned}
& \int_{0}^{T}\left(\left\|\nabla^{2} u\right\|_{L^{2}}^{2}+\left\|\nabla^{2} u\right\|_{L^{q}}^{\frac{q+1}{q}}+t\left\|\nabla^{2} u\right\|_{L^{2} \cap L^{q}}^{2}\right) \mathrm{d} t \\
& +\int_{0}^{T}\left(\|\nabla p\|_{L^{2}}^{2}+\|\nabla p\|_{L^{q}}^{\frac{q+1}{q}}+t\|\nabla p\|_{L^{2} \cap L^{q}}^{2}\right) \mathrm{d} t \leq C,
\end{aligned}
$$

which combined with (3.1) and (3.45) gets (3.37). The Lemma 3.5 is proved.

Lemma 3.6 (see [13]) There exists a positive constant $C$ depending on $T$ such that for $q>2$,

$$
\sup _{t \in[0, T]}\left\|\rho \bar{x}^{a}\right\|_{L^{1} \cap H^{1} \cap W^{1, q}} \leq C(T) .
$$

Proof. First, setting $\rho=\rho \bar{x}^{a}$ in $(1.1)_{1}$ that satisfies

$$
\partial_{t}\left(\rho \bar{x}^{a}\right)+u \cdot \nabla\left(\rho \bar{x}^{a}\right)-a \rho \bar{x}^{a} u \cdot \nabla \log \bar{x}=0 .
$$

Next, we can take the $x_{i}$-derivative on both sides of the (3.51) finds

$$
\begin{aligned}
0= & \partial_{t} \partial_{i}\left(\rho \bar{x}^{a}\right)+u \cdot \nabla \partial_{i}\left(\rho \bar{x}^{a}\right)+\partial_{i} u \cdot \nabla\left(\rho \bar{x}^{a}\right)-a \partial_{i}\left(\rho \bar{x}^{a}\right) u \cdot \nabla \log \bar{x} \\
& -a \rho \bar{x}^{a} \partial_{i} u \cdot \nabla \log \bar{x}-a \rho \bar{x}^{a} u \cdot \partial_{i} \nabla \log \bar{x} .
\end{aligned}
$$

the (3.52) multiplied by $\left|\nabla\left(\rho \bar{x}^{a}\right)\right|^{r-2} \partial_{i}\left(\rho \bar{x}^{a}\right)$ and integrating the resulting equality by parts over $\mathbb{R}^{2}$, and then for any $r \in[2, q]$, we obtain that

$$
\begin{aligned}
\frac{\mathrm{d}}{\mathrm{d} t}\left\|\nabla\left(\rho \bar{x}^{a}\right)\right\|_{L^{r}} \leq & C\left(1+\|u \cdot \nabla \log \bar{x}\|_{L^{\infty}}+\|\nabla u\|_{L^{\infty}}\right)\left\|\nabla\left(\rho \bar{x}^{a}\right)\right\|_{L^{r}} \\
& +C\left\|\rho \bar{x}^{a}\right\|_{L^{\infty}}\left(\|u\| \nabla^{2} \log \bar{x}\left\|_{L^{r}}+\right\| \nabla u\|\nabla \log \bar{x}\|_{L^{r}}\right) \\
\leq & C\left(1+\|\nabla u\|_{W^{1, q}}\right)\left\|\nabla\left(\rho \bar{x}^{a}\right)\right\|_{L^{r}} \\
& +C\left\|\rho \bar{x}^{a}\right\|_{L^{\infty}}\left(\|\nabla u\|_{L^{r}}+\left\|u \bar{x}^{-\frac{3}{5}}\right\|_{L^{4 r}}\left\|\bar{X}^{-\frac{4}{3}}\right\| \frac{4 r}{3}_{L^{3}}\right) \\
\leq & C\left(1+\left\|\nabla\left(\rho \bar{x}^{a}\right)\right\|_{L^{r}}+\left\|\nabla\left(\rho \bar{x}^{a}\right)\right\|_{L^{q}}\right)\left(1+\|\nabla u\|_{W^{1, q}}\right) .
\end{aligned}
$$

For the second and the last inequalities of (3.53), it has used (3.35) and (3.31), respectively. Setting $r=q$ in (3.53), and applying Gronwall's inequality along with (3.37) indicates that

$$
\sup _{t \in[0, T]}\left\|\nabla\left(\rho \bar{x}^{a}\right)\right\|_{L^{q}} \leq C .
$$

And choosing $r=2$ in (3.53), we will deduce from (3.37) and (3.54) that 


$$
\sup _{t \in[0, T]}\left\|\nabla\left(\rho \bar{x}^{a}\right)\right\|_{L^{2}} \leq C
$$

Combining (3.54) with (3.31) gives (3.50). The Lemma 3.6 is proved.

Lemma 3.7 There exists a positive constant $C$ such that

$$
\begin{gathered}
\sup _{t \in[0, T] \|}\left\|\theta \bar{x}^{\frac{a}{2}}\right\|_{L^{2}}^{2}+\int_{0}^{T}\left\|\nabla \theta \bar{x}^{\frac{a}{2}}\right\|_{L^{2}}^{2} \mathrm{~d} t \leq C(T), \\
\sup _{t \in[0, T]}\left(t\left\|\nabla \theta \bar{x}^{\frac{a}{2}}\right\|_{L^{2}}^{2}\right)+\int_{0}^{T} t\left\|\Delta \theta \bar{x}^{\frac{a}{2}}\right\|_{L^{2}}^{2} \mathrm{~d} t \leq C(T) .
\end{gathered}
$$

Proof. The $(1.1)_{3}$ multiplied by $\theta \bar{x}^{a}$ and integrating the resulting equality by parts over $\mathbb{R}^{2}$, we have

$$
\begin{aligned}
& \frac{1}{2} \frac{\mathrm{d}}{\mathrm{d} t}\left\|\theta \bar{x}^{\frac{a}{2}}\right\|_{L^{2}}^{2}+\kappa\left\|\nabla \theta \bar{x}^{\frac{a}{2}}\right\|_{L^{2}}^{2} \\
& =\frac{\kappa}{2} \int|\theta|^{2} \Delta \bar{x}^{a} \mathrm{~d} x+\frac{1}{2} \int|\theta|^{2} u \cdot \nabla \bar{x}^{a} \mathrm{~d} x \triangleq \hat{M}_{1}+\hat{M}_{2},
\end{aligned}
$$

where

$$
\begin{aligned}
\hat{M}_{1} & =\frac{\kappa}{2} \int|\theta|^{2} \Delta \bar{x}^{a} \mathrm{~d} x \\
& \leq C \int|\theta|^{2} \bar{x}^{a} \bar{x}^{-2} \log ^{4}\left(e+|x|^{2}\right) \mathrm{d} x \\
& \leq C\left\|\theta \bar{x}^{\frac{a}{2}}\right\|_{L^{2}}^{2}, \\
\hat{M}_{2} & =\frac{1}{2} \int|\theta|^{2} u \cdot \nabla \bar{x}^{a} \mathrm{~d} x \\
& \leq C\left\|\theta \bar{x}^{\frac{a}{2}}\right\|_{L^{4}}\left\|\theta \bar{x}^{\frac{a}{2}}\right\|_{L^{2}}\left\|u \bar{x}^{-\frac{3}{4}}\right\|_{L^{4}} \\
& \leq C\left\|\theta \bar{x}^{\frac{a}{2}}\right\|_{L^{2}}^{2}+\frac{\kappa}{2}\left\|\nabla \theta \bar{x}^{\frac{a}{2}}\right\|_{L^{2}}^{2} .
\end{aligned}
$$

Substituting (3.59), (3.60) into (3.58), we get

$$
\frac{1}{2} \frac{\mathrm{d}}{\mathrm{d} t}\left\|\theta \bar{x}^{\frac{a}{2}}\right\|_{L^{2}}^{2}+\frac{\kappa}{2}\left\|\nabla \theta \bar{x}^{\frac{a}{2}}\right\|_{L^{2}}^{2} \leq C\left\|\theta \bar{x}^{\frac{a}{2}}\right\|_{L^{2}}^{2} .
$$

Using Gronwall's inequality to (3.61), we obtain (3.56).

Next, we will estimate the (3.57). The (1.1) $)_{3}$ Multiplied by $\Delta \theta \bar{x}^{a}$ and integration by parts over $\mathbb{R}^{2}$, we find

$$
\begin{aligned}
& \frac{1}{2} \frac{\mathrm{d}}{\mathrm{d} t}\left\|\nabla \theta \bar{x}^{\frac{a}{2}}\right\|_{L^{2}}^{2}+\kappa\left\|\Delta \theta \bar{x}^{\frac{a}{2}}\right\|_{L^{2}}^{2} \\
& \leq C \int|\nabla u||\nabla \theta|^{2} \bar{x}^{a} \mathrm{~d} x+C \int|u||\nabla \theta|^{2}\left|\nabla \bar{x}^{a}\right| \mathrm{d} x+C \int|\nabla \theta||\Delta \theta|\left|\nabla \bar{x}^{a}\right| \mathrm{d} x \\
& \triangleq \sum_{i=1}^{3} \tilde{M}_{i},
\end{aligned}
$$


where

$$
\begin{aligned}
& \tilde{M}_{1}=C \int|\nabla u||\nabla \theta|^{2} \bar{x}^{a} \mathrm{~d} x \\
& \leq C\|\nabla u\|_{L^{\infty}}\left\|\nabla \theta \bar{x}^{\frac{a}{2}}\right\|_{L^{2}}^{2} \\
& \leq C\|\nabla u\|_{L^{2}}^{\frac{q-2}{2(q-1)}}\left\|\nabla^{2} u\right\|_{L^{q}}^{\frac{q-2}{2(q-1)}}\left\|\nabla \theta \bar{X}^{\frac{a}{2}}\right\|_{L^{2}}^{2} \\
& \leq C\left(1+\left\|\nabla^{2} u\right\|_{L^{q}}^{\frac{q+1}{q}}\right)\left\|\nabla \theta \bar{X}^{\frac{a}{2}}\right\|_{L^{2}}^{2}, \\
& \tilde{M}_{2}=C \int|u||\nabla \theta|^{2}\left|\nabla \bar{x}^{a}\right| \mathrm{d} x \\
& \leq C\left\||\nabla \theta|^{2-\frac{2}{3 a}} \bar{x}^{a-\frac{1}{3}}\right\|_{L^{6 a-2}}\left\|u \bar{x}^{-\frac{1}{3}}\right\|_{L^{6 a}}\left\|\left.\nabla \theta\right|^{\frac{2}{3 a}}\right\|_{L^{6 a}} \\
& \leq C\left\|\nabla \theta \bar{x}^{\frac{a}{2}}\right\|_{L^{2}}^{\frac{6 a-2}{3 a}}\|\nabla \theta\|_{L^{4}}^{\frac{2}{3 a}} \\
& \leq C\left\|\nabla \theta \bar{x}^{\frac{a}{2}}\right\|_{L^{2}}^{2}+C\|\nabla \theta\|_{L^{4}}^{2} \\
& \leq C\left\|\nabla \theta \bar{x}^{\frac{a}{2}}\right\|_{L^{2}}^{2}+\frac{\kappa}{4}\left\|\Delta \theta \bar{x}^{\frac{a}{2}}\right\|_{L^{2}}^{2}, \\
& \tilde{M}_{3}=C \int\left|\nabla \theta\left\|\Delta \theta|| \nabla \bar{X}^{a} \mid \mathrm{d} x \leq \frac{\kappa}{4}\right\| \Delta \theta \bar{X}^{\frac{a}{2}}\left\|_{L^{2}}^{2}+C\right\| \nabla \theta \bar{X}^{\frac{a}{2}} \|_{L^{2}}^{2} .\right.
\end{aligned}
$$

Submitting $\tilde{M}_{1}, \tilde{M}_{2}, \tilde{M}_{3}$ into (3.62), one has

$$
\frac{1}{2} \frac{\mathrm{d}}{\mathrm{d} t}\left\|\nabla \theta \bar{x}^{\frac{a}{2}}\right\|_{L^{2}}^{2}+\frac{\kappa}{2}\left\|\Delta \theta \bar{x}^{\frac{a}{2}}\right\|_{L^{2}}^{2} \leq C\left(1+\left\|\nabla^{2} u\right\|_{L^{q}}^{\frac{q+1}{q}}\right)\left\|\nabla \theta \bar{x}^{\frac{a}{2}}\right\|_{L^{2}}^{2} .
$$

Multiplying (3.66) by $t$, and togethering with (3.56) and (3.37), then employing Gronwall's inequlity, one obtains the (3.57). This completes the Lemma 3.7.

Lemma 3.8 There exists a positive constant $C$ such that

$$
\sup _{t \in[0, T]} t\left(\left\|\sqrt{\rho} u_{t}\right\|_{L^{2}}^{2}+\left\|\theta_{t}\right\|_{L^{2}}^{2}+\left\|\nabla^{2} \theta\right\|_{L^{2}}^{2}\right)+\int_{0}^{T}\left(t\left\|\nabla u_{t}\right\|_{L^{2}}^{2}+t\left\|\nabla \theta_{t}\right\|_{L^{2}}^{2}\right) \mathrm{d} t \leq C(T) \text {. }
$$

Proof. For any $\eta \in(0,1]$ and any $s>2$, it deduces from (3.40), (3.35) that

$$
\left\|\rho^{\eta} u\right\|_{L^{s / \eta}}+\left\|u \bar{x}^{-\eta}\right\|_{L^{s / \eta}} \leq C .
$$

Next, it will prove that

$$
\sup _{t \in[0, T]}\left(\|\nabla u\|_{L^{2}}^{2}+\|\nabla \theta\|_{L^{2}}^{2}\right)+\int_{0}^{T}\left(\left\|\sqrt{\rho} u_{t}\right\|_{L^{2}}^{2}+\left\|\theta_{t}\right\|_{L^{2}}^{2}+\|\Delta \theta\|_{L^{2}}^{2}\right) \mathrm{d} t \leq C .
$$

With (3.2) at hand, we need only to show

$$
\int_{0}^{T}\left(\left\|\sqrt{\rho} u_{t}\right\|_{L^{2}}^{2}+\left\|\theta_{t}\right\|_{L^{2}}^{2}\right) \mathrm{d} t \leq C
$$

First, it is easy to show that 


$$
\begin{aligned}
\left\|\sqrt{\rho} u_{t}\right\|_{L^{2}}^{2} & \leq\|\sqrt{\rho} \dot{u}\|_{L^{2}}^{2}+\|\sqrt{\rho} \mid u\| \nabla u\|\|_{L^{2}}^{2} \\
& \leq\|\sqrt{\rho} \dot{u}\|_{L^{2}}^{2}+C\|\sqrt{\rho} u\|_{L^{2}}^{2}\|\nabla u\|_{L^{3}}^{2} \\
& \leq\|\sqrt{\rho} \dot{u}\|_{L^{2}}^{2}+C\|\nabla u\|_{L^{2}}^{2}+C\left\|\nabla^{2} u\right\|_{L^{2}}^{2} .
\end{aligned}
$$

Then, due to (2.1) and (3.68), we can combine (2.1), (3.2) with (1.1) $)_{3}$ gives

$$
\left\|\theta_{t}\right\|_{L^{2}}^{2} \leq C\|\Delta \theta\|_{L^{2}}^{2}+\|u\| \nabla \theta\left\|_{L^{2}}^{2} \leq C\right\| \Delta \theta\left\|_{L^{2}}^{2}+\right\| \nabla \theta \bar{x}^{\frac{a}{2}} \|_{L^{2}}^{2},
$$

It has used the following facts about (3.72) of the last inequality

$$
\|u\| \nabla \theta\left\|_{L^{2}}^{2} \leq C\right\| u \bar{x}^{-\frac{a}{4}}\left\|_{L^{8}}^{4}\right\| \nabla \theta\left\|_{L^{4}}^{2}+C\right\| \nabla \theta \bar{x}^{\frac{a}{2}}\left\|_{L^{2}}^{2} \leq \frac{1}{2}\right\| \nabla^{2} \theta\left\|_{L^{2}}^{2}+C\right\| \nabla \theta \bar{x}^{\frac{a}{2}} \|_{L^{2}}^{2} .
$$

According to (3.68) and (2.1), we can give (3.70) by the combination of (3.71), (3.72), (3.37), and (3.56).

Next, differentiating $(1.1)_{2}$ with respect to $t$ shows

$$
\rho u_{t t}+\rho u \cdot \nabla u_{t}-\mu \Delta u_{t}+\nabla p_{t}=-\rho_{t}\left(u_{t}+u \cdot \nabla u\right)-\rho u_{t} \cdot \nabla u .
$$

(3.74) multiplied by $u_{t}$ and integration by parts over $\mathbb{R}^{2}$, it deduces from $(1.1)_{1}$ and $(1.1)_{4}$ that

$$
\begin{aligned}
& \frac{1}{2} \frac{\mathrm{d}}{\mathrm{d} t} \int \rho\left|u_{t}\right|^{2} \mathrm{~d} x+\mu \int\left|\nabla u_{t}\right|^{2} \mathrm{~d} x \\
& \leq C \int \rho|u|\left|u_{t}\right|\left(\nabla u_{t}+|u|\left|\nabla^{2} u\right|+|\nabla u|^{2}\right) \mathrm{d} x \\
& \quad+C \int \rho|u|^{2}|\nabla u|\left|\nabla u_{t}\right| \mathrm{d} x+C \int \rho\left|u_{t}\right|^{2}|\nabla u| \mathrm{d} x \\
& \triangleq \sum_{i=1}^{3} \bar{M}_{i},
\end{aligned}
$$

where

$$
\begin{aligned}
& \bar{M}_{1} \leq C\|\sqrt{\rho} u\|_{L^{6}}\left\|\sqrt{\rho} u_{t}\right\|_{L^{2}}^{\frac{1}{2}}\left\|\sqrt{\rho} u_{t}\right\|_{L^{6}}^{\frac{1}{2}}\left(\left\|\nabla u_{t}\right\|_{L^{2}}+\|\nabla u\|_{L^{4}}^{2}\right) \\
&+C\left\|\rho^{\frac{1}{4}} u\right\|_{L^{12}}^{2}\left\|\sqrt{\rho} u_{t}\right\|_{L^{2}}^{\frac{1}{2}}\left\|\sqrt{\rho} u_{t}\right\|_{L^{6}}^{\frac{1}{2}}\left\|\nabla^{2} u\right\|_{L^{2}} \\
& \leq C\left\|\sqrt{\rho} u_{t}\right\|_{L^{2}}^{\frac{1}{2}}\left(\left\|\sqrt{\rho} u_{t}\right\|_{L^{2}}+\left\|\nabla u_{t}\right\|_{L^{2}}\right)^{\frac{1}{2}}\left(\left\|\nabla u_{t}\right\|_{L^{2}}+\left\|\nabla^{2} u\right\|_{L^{2}}\right) \\
& \leq \frac{\mu}{4}\left\|\nabla u_{t}\right\|_{L^{2}}^{2}+C\left(1+\left\|\sqrt{\rho} u_{t}\right\|_{L^{2}}^{2}+\left\|\nabla^{2} u\right\|_{L^{2}}^{2}\right), \\
& \bar{M}_{2}+\bar{M}_{3} \leq C\|\sqrt{\rho} u\|_{L^{8}}^{2}\|\nabla u\|_{L^{4}}\left\|\nabla u_{t}\right\|_{L^{2}}+\|\nabla u\|_{L^{2}}\left\|\sqrt{\rho} u_{t}\right\|_{L^{6}}^{\frac{3}{2}}\left\|\sqrt{\rho} u_{t}\right\|_{L^{2}}^{\frac{1}{2}} \\
& \leq \frac{\mu}{4}\left\|\nabla u_{t}\right\|_{L^{2}}^{2}+C\left(1+\left\|\sqrt{\rho} u_{t}\right\|_{L^{2}}^{2}+\left\|\nabla^{2} u\right\|_{L^{2}}^{2}\right) .
\end{aligned}
$$

Submitting $\bar{M}_{1}, \bar{M}_{2}+\bar{M}_{3}$ into (3.75) gives

$$
\frac{\mathrm{d}}{\mathrm{d} t}\left\|\sqrt{\rho} u_{t}\right\|_{L^{2}}^{2}+\mu\left\|\nabla u_{t}\right\|_{L^{2}}^{2} \leq C\left\|\sqrt{\rho} u_{t}\right\|_{L^{2}}^{2}+C\left(\left\|\nabla^{2} u\right\|_{L^{2}}^{2}+1\right) .
$$


Then, we multiply (3.78) by $t$, and link to Gronwall's inequality and (3.37) lead to

$$
\sup _{t \in[0, T]} t\left\|\sqrt{\rho} u_{t}\right\|_{L^{2}}^{2}+\int_{0}^{T} t\left\|\nabla u_{t}\right\|_{L^{2}}^{2} \mathrm{~d} t \leq C .
$$

Next, differentiating $(1.1)_{3}$ with respect to $t$ show

$$
\theta_{t t}+u_{t} \cdot \nabla \theta+u \cdot \nabla \theta_{t}-\kappa \Delta \theta_{t}=0
$$

Now, the (3.80) multiplied by $\theta_{t}$ and integration by parts over $\mathbb{R}^{2}$, we find

$$
\begin{aligned}
& \frac{1}{2} \frac{\mathrm{d}}{\mathrm{d} t} \int\left|\theta_{t}\right|^{2} \mathrm{~d} x+\kappa \int\left|\nabla \theta_{t}\right|^{2} \mathrm{~d} x \\
& =\int u_{t} \cdot \nabla \theta_{t} \cdot \theta \mathrm{d} x \\
& \leq C\left\|\theta \bar{x}^{\frac{a}{2}}\right\|_{L^{\frac{8 a}{a-1}}}\left\|u_{t} \bar{x}^{-\frac{2 a-1}{4}}\right\|_{L^{8 a}}\left\|\nabla \theta_{t}\right\|_{L^{2}} \\
& \leq \frac{\mu}{2}\left(\left\|\nabla u_{t}\right\|_{L^{2}}^{2}+\left\|\sqrt{\rho} u_{t}\right\|_{L^{2}}^{2}\right)+\frac{\kappa}{2}\left\|\nabla \theta_{t}\right\|_{L^{2}}^{2} .
\end{aligned}
$$

Next, the (3.81) multiplied by $t$ and integration by parts over $[0, T]$, and due to (3.70), we have

$$
\sup _{t \in[0, T]} t\left\|\theta_{t}\right\|_{L^{2}}^{2}+\frac{\kappa}{2} \int_{0}^{T} t\left\|\nabla \theta_{t}\right\|_{L^{2}}^{2} \mathrm{~d} t \leq \frac{\mu}{2} \int_{0}^{T} t\left\|\nabla u_{t}\right\|_{L^{2}}^{2}+C .
$$

Finally, it follows from $(1.1)_{3}$, and (3.73) that

$$
\begin{aligned}
\left\|\nabla^{2} \theta\right\|_{L^{2}}^{2} & \leq C\left\|\theta_{t}\right\|_{L^{2}}^{2}+C\|u\| \nabla \theta \|_{L^{2}}^{2} \\
& \leq C\left\|\theta_{t}\right\|_{L^{2}}^{2}+\frac{1}{2}\left\|\nabla^{2} \theta\right\|_{L^{2}}^{2}+C\left\|\nabla \theta \bar{x}^{\frac{a}{2}}\right\|_{L^{2}}^{2},
\end{aligned}
$$

which combine with (3.57), (3.79) and (3.82) gains (3.67). Finally, the proof of Lemma 3.8 is finished.

\subsection{Proof of Theorem 1.1}

In this section, we will give the proof of Theorem 1.1.

Proof: According to Lemmas 3.1-3.8, using standard theory of local existence, It assumes that there is a $T_{*}>0$ such that systems (1.1) and (1.2) have a local and unique strong solution $(\rho, u, p, \theta)$ on $\mathbb{R}^{2} \times\left(0, T_{*}\right]$. Next, we will extend the local solution to all time.

Set

$$
T^{*}=\sup \left\{T \mid(\rho, u, p, \theta) \text { is a strong solution on } \mathbb{R}^{2} \times(0, T]\right\} .
$$

It deduces from (3.67), for any $0<\tau<T<T^{*}$ with $T$ finite, and any $q \geq 2$ that,

$$
\nabla u, \nabla \theta, \theta \in C\left([\tau, T] ; L^{2} \cap L^{q}\right) .
$$

Then, along with standard embedding

$$
L^{\infty}\left(\tau, T ; H^{1}\right) \cap H^{1}\left(\tau, T ; L^{2}\right) \hookrightarrow C\left([\tau, T] ; L^{q}\right) \text {, for any } q \in[2, \infty) .
$$


And, due to (3.36), (3.49), and ([23] Lemma 2.3) we have

$$
\rho \in C\left([0, T] ; L^{1} \cap H^{1} \cap W^{1, q}\right) .
$$

we declare that

$$
T^{*}=\infty .
$$

On the contrary, if $T^{*}<\infty$, it deduces from (4.2), (4.2), (3.2), (3.6), (3.49), and (3.50) that

$$
(\rho, u, \theta)\left(x, T^{*}\right)=\lim _{t \rightarrow T^{*}}(\rho, u, \theta)(x, t) .
$$

conforms to the initial condition (1.6) at $t=T$. So, we can assume the initial data is the $(\rho, u, \theta)\left(x, T^{*}\right)$, since the existence and uniqueness of local strong solutions signifies that there is a some $T^{* *}>T^{*}$, such that Theorem (1.1) holds for $T=T^{* *}$. This is contradictory with the hypothesis of $T^{*}$ in (3.84), so the (3.87) holds. Hence, Lemmas 3.1-3.8 and the local existence and uniqueness of strong solutions indicate that $(\rho, u, p, \theta)$ is actually the unique strong solution on $\mathbb{R}^{2} \times[0, T]$ for any $0<T<T^{*}=\infty$. This completes the proof of Theorem 1.1 .

\section{Conflicts of Interest}

The author declares no conflicts of interest regarding the publication of this paper.

\section{References}

[1] Majda, A. (2003) Introduction to PDEs and Waves for the Atmosphere and Ocean. Courant Lecture Notes in Mathematics, Vol. 9, AMS/CIMS. https://doi.org/10.1090/cln/009

[2] Li, H.P. (2013) Research on Related Problems of Boussinesq Equations with Nonlinear Diffusion. Jilin University, Changchun.

[3] Teman, R. (1977) Navier-Stokes Equations: Theory and Numerical Analysis (Studies in Mathematics and Its Applications). Elsevier, Amsterdam.

[4] Lions, P.L. (1996) Mathematical Topics in Fluid Mechanics, Vol. I: Incompressible Models. Oxford University Press, Oxford.

[5] Simon, J. (1990) Nonhomogeneous Viscous Incompressible Fluids: Existence of Velocity, Density, and Pressure. SIAM Journal on Mathematical Analysis, 21, 1093-1117. https://doi.org/10.1137/0521061

[6] Majda, A.J. and Bertozzi, A.L. (2001) Vorticity and Incompressible Flow. Cambridge University Press, Cambridge. https://doi.org/10.1017/CBO9780511613203

[7] Antontsev, S.A., Kazhikov, A.V. and Monakhov, V.N. (1990) Boundary Value Problems in Mechanics of Nonhomogeneous Fluids. North-Holland Publishing Co., Amsterdam.

[8] Lorca, S.A. and Boldrini, J.L. (1996) The Initial Value Problem for a Generalized Boussinesq Model: Regularity and Global Existence of Strong Solutions. Matemática Contemporânea, 11, 71-94.

[9] Lorca, S.A. and Boldrini, J.L. (1999) The Initial Value Problem for a Generalized Boussinesq Model. Nonlinear Analysis, 36, 457-480. 
https://doi.org/10.1016/S0362-546X(97)00635-4

[10] Qiu, H. and Yao, Z. (2017) Well-Posedness for Density-Dependent Boussinesq Equations without Dissipation Terms in Besov Spaces. Computers \& Mathematics with Applications, 73, 1920-1931. https://doi.org/10.1016/j.camwa.2017.02.041

[11] Zhang, Z. (2016) 3D Density-Dependent Boussinesq Equations with Velocity Field in BMO Spaces. Acta Applicandae Mathematicae, 142, 1-8. https://doi.org/10.1007/s10440-015-0011-8

[12] Lü, B.Q., Shi, X.D. and Zhong, X. (2018) Global Existence and Large Time Asymptotic Behavior of Strong Solutions to the Cauchy Problem of 2D Density-Dependent Navier-Stokes Equations with Vacuum. Nonlinearity, 31, 2617-2632. https://doi.org/10.1088/1361-6544/aab31f

[13] Lü, B.Q. and Huang, B. (2015) On Strong Solutions to the Cauchy Problem of the Two-Dimensional Compressible Magnetohydrodynamic Equations with Vacuum. Nonlinearity, 28, 509-530. https://doi.org/10.1088/0951-7715/28/2/509

[14] Lü, B.Q., Xu, Z.H. and Zhong, X. (2017) Global Existence and Large Time Asymptotic Behavior of Strong Solution to the Cauchy Problem of 2D Density-Dependent Magnatohydrodynamic Equations with Vacuum. Journal de Mathématiques Pures et Appliquées, 108, 41-62. https://doi.org/10.1016/j.matpur.2016.10.009

[15] Li, J. and Xin, Z.P. (2019) Global Well-Posedness and Large Time Asymptotic Behavior of Classical Solutions to the Compressible Navier-Stokes Equations with Vacuum. Annals of PDE, 5, 1-37. https://doi.org/10.1007/s40818-019-0064-5

[16] Hoff, D. (1995) Global Solutions of the Navier-Stokes Equations for Multidimensional Compressible Flow with Discontinuous Initial Data. Journal of Differential Equations, 120, 215-154. https://doi.org/10.1006/jdeq.1995.1111

[17] Lü, B.Q., Shi, X.D. and Xu, X.Y. (2016) Global Well-Posedness and Large Time Asymptotic Behavior of Strong Solutions to the Compressible Magnetohydrodynamic Equations with Vacuum. Indiana University Mathematics Journal, 65, 925-975. https://doi.org/10.1512/iumj.2016.65.5813

[18] Li, J. and Liang, Z.L. (2014) On Local Classical Solutions to the Cauchy Problem of the Two-Dimensional Barotropic Compressible Navier-Stokes Equations with Vacuum. Journal de Mathématiques Pures et Appliquées, 102, 640-671. https://doi.org/10.1016/j.matpur.2014.02.001

[19] Huang, X.D., Li, J. and Xin, Z.P. (2012) Global Well-Posedness of Classical Solutions with Large Oscillations and Vacuum to the Three-Dimensional Isentropic Compressible Navier-Stokes Equations. Communications on Pure and Applied Mathematics, 65, 549-585. https://doi.org/10.1002/cpa.21382

[20] Liu, S.Q. and Zhang, J.W. (2016) Global Well-Posedeness for the Two-Dimensional Equations of Nonhomogeneous Incompressible Liquid Crystal Flows with Nonnegative Density. Discrete and Continuous Dynamical Systems-Series B, 21, 2631-2648. https://doi.org/10.3934/dcdsb.2016065

[21] Huang, X.D. and Wang, Y. (2014) Global Strong Solution with Vacuum to the Two-Dimensional Density-Dependent Navier-Stokes System. SIAM Journal on Mathematical Analysis, 46, 1771-1788. https://doi.org/10.1137/120894865

[22] Ishimura, N. and Morimoto, H. (1999) Remarks on the Blow-Up Criterion for the 3D Boussinesq Equations. Mathematical Models and Methods in Applied Sciences, 9, 1323-1332. https://doi.org/10.1142/S0218202599000580

[23] Liang, Z. (2015) Local Strong Solution and Blow-Up Criterion for the 2D Nonhomogeneous Incompressible Fluids. Journal of Differential Equations, 258, 2633-2654. https://doi.org/10.1016/j.jde.2014.12.015 
[24] Stein, E.M. (1993) Harmonic Analysis: Real-Variable Methods, Orthogonality, and Oscillatory Integrals. Princeton University Press, Princeton.

[25] Coifman, R., Lions, P.L., Meyer, Y. and Semmes, S. (1993) Compensated Compactness and Hardy Spaces. Journal de Mathématiques Pures et Appliquées, 72, 247-286.

[26] Temam, R. (2001) Navier-Stokes Equations: Theory and Numerical Analysis. AMS Chelsea Publishing, Providence.

[27] Desjardins, B. (1997) Regularity Results for Two-Dimensional Flows of Multiphase Viscous Fluids. Archive for Rational Mechanics and Analysis, 137, 135-158.

https://doi.org/10.1007/s002050050025 